\title{
Left Sided Acute Appendicitis: Radiological Aspects
}

\author{
Kassim Sidibé1 ${ }^{*}$, Pierlesky Elion Ossibi², Zacharia Traoré1, Imane Kamaoui ${ }^{3}$, \\ Youssef Lamrani' ${ }^{1}$, Meryem Boubbou ${ }^{1}$, Moustapha Maâroufi ${ }^{1}$, \\ Abdoul Aliou Zabeirou Oudou'2, Said Ait Laalim², Khalid Mazaz², Siham Tizniti1 \\ ${ }^{1}$ Department of Radiology, Hassan II Teaching Hospital, Fes, Morocco \\ ${ }^{2}$ Department of Visceral Surgery, Hassan II Teaching Hospital, Fes, Morocco \\ ${ }^{3}$ Department of Radiology, Mohammed VI Teaching Hospital, Oujda, Morocco \\ Email: *kadiatoulakas@yahoo.fr
}

Received 30 December 2015; accepted 31 May 2016; published 3 June 2016

Copyright (C) 2016 by authors and Scientific Research Publishing Inc.

This work is licensed under the Creative Commons Attribution International License (CC BY). http://creativecommons.org/licenses/by/4.0/

(c) (i) Open Access

\section{Abstract}

Left sided appendicitis is a rare pathology. Two situations may explain the occurrence of the disease: situs inversus or midgut malrotation. Its diagnosis is based on clinical presentation but confirmed by radiological examination. Our observation is based on a left-sided acute appendicitis with a midgut malrotation in a 31-year-old patient with no clinical history.

\section{Keywords}

\section{Appendicitis, Left, Midgut Rotation, Radiological Aspects}

\section{Introduction}

Acute appendicitis constitutes the commonest abdominal surgical emergency. Typically acute appendicitis presents as a sharp abdominal pain in the right iliac fossa (RIF) or sometimes starting in the epigastric region, continuous in nature without irradiations sometimes associated with nausea and vomiting. Abdominal examination may reveal RIF tenderness more prominent at the Mc Burney point. Abdominal pain could be exacerbated by abrupt removal of abdominal pressure, hence a rebound tenderness, at the RIF (Blumberg sign) or elicited by pressing on the left lower quadrant indicating a positive Rovsing's sign. Histologically an inflammation of the appendiceal wall: the presence of neutrophils in the mucosa and the muscularis propria with an ulceration of the mucosa could be observed. The presence of an appendicular syndrome at the left iliac fossa is an extremely rare occurrence. This could however be possible as a result of either a complete situs inversus or could even be

${ }^{*}$ Corresponding author.

How to cite this paper: Sidibé, K., Ossibi, P.E., Traoré, Z., Kamaoui, I., Lamrani, Y., Boubbou, M., Maâroufi, M., Oudou, A.A.Z., Laalim, S.A., Mazaz, K. and Tizniti, S. (2016) Left Sided Acute Appendicitis: Radiological Aspects. Open Journal of Radiology, 6, 96-99. http://dx.doi.org/10.4236/ojrad.2016.62014 
explained by the presence a common mesentery and could lead to a misdiagnosis. Imaging allows precise positive diagnosis of the lesion thereby guiding its surgical management. We report the case of a patient who presented with acute appendicitis left side of a complete common mesentery.

\section{Case Report}

Patient, 31 years old without significant history was admitted to our unit with left iliac fossa pain and fever. Symptoms dated back to 48 hours prior to his admission with the onset of abdominal pain associated with a few episodes of vomiting.

Physical examination found a conscious patient febrile $38.5^{\circ} \mathrm{C}$ and heart rate 92. Abdominal examination revealed tenderness in the left iliac fossa.

Laboratory tests came back with $14,500 / \mathrm{mm}^{3}$ leukocytosis and C-reactive protein level at $178 \mathrm{mg} / \mathrm{l}$.

Abdominal ultrasound showed significant infiltration of the surrounding fat in the left iliac fossa.

Complementary abdominopelvic CT (multibaret scanner, spiral acquisition, $5 \mathrm{~mm}$ slice thickness $2.5 \mathrm{~mm}$ reconstructions) with intravenous iodinated contrast medium made evident an entire colic loop shifted to the left side of the abdominal cavity suggestive of a common mesentery (Figure 1) and an inflamed appendix, thickened and medially situated with infiltration of its surrounding fat (Figure 2 and Figure 3).

Patient was admitted to emergency theatre where laparotomy and subsequent appendectomy was performed.

The postoperative recovery was uneventful and patient was discharged 2 days after emergent surgery with favorable outcome.

\section{Discussion}

Birth defects of gastrointestinal tract constitute a frequent morbidity in children and a rather less common pathology in adults [1]. These birth defects include atresia of the small intestine and colon, abnormal rotation and fixation of the primitive gut, anorectal abnormalities, and intestinal duplications. Normally, after complete rotation during intra uterine life, the mesentery has a large base stretching from the left upper quadrant down to the right iliac fossa.

Intestinal malrotation is a defectin or an incomplete rotation of the mid gut that results in a shortened mesentery with a displaced Treitz ligament and cecum [1]. This rotation defect complicates with mid gut volvulus in case malrotation stopsat $180^{\circ}$ [2] or could be asymptomatic if it stops at 90 giving rise to a complete common mesentery which rarely complicates hence explaining its fortuitous discovery on radiological imaging done for other indications.

Embryologically, a common mesentery results from persistence:

- of the primitive gut which fails to undergo a normal rotation;

- of a posterior attachment to the primitive gut wall after 12 weeks, during reintegration of the latter in the abdomen.

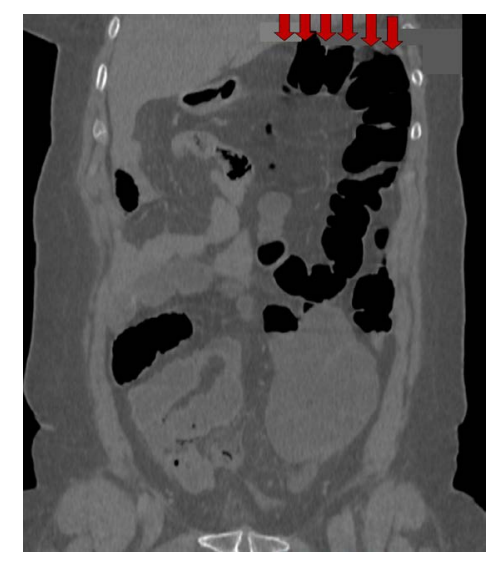

Figure 1. Abdominal CT coronal section after contrast medium injection revealing the entire colic loop on the left side of the abdominal cavity evoking a common mesentery. 


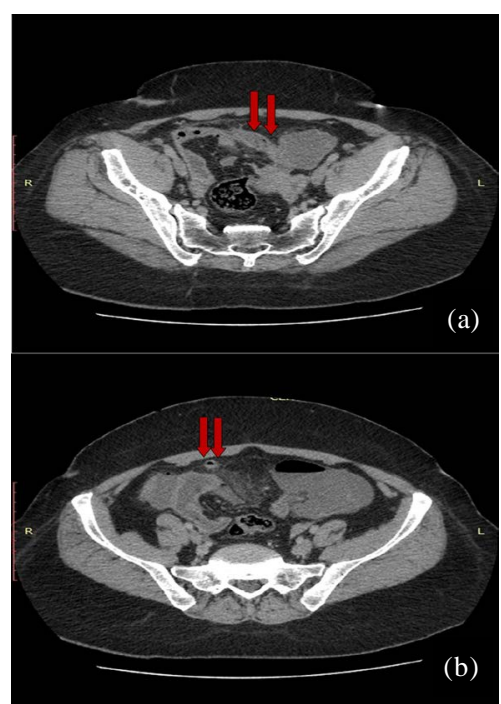

Figure 2. Axial reconstruction after injection of contrast medium showing the abdominal cavity: (a) thickened inflamed appendix closely linked with cecum in the LIF with infiltration of surrounding fat; (b) the inflamed appendiceal tip sitting next to the rectus abdominis muscle.

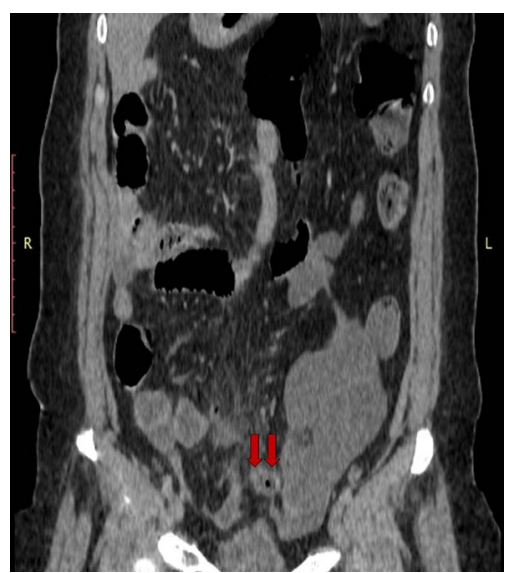

Figure 3. Abdominal CT after contrast medium injection revealing an inflamed appendix slightly detached from caecum as well as thickening of the surrounding fat.

Appendicitis accounts for almost a third of acute abdomens yet the presence of an appendix on the left represents an extremelyrare occurrence [3]. A recent review of literature attributed 63 cases reported to situs inversus [4] [5]. Cases associated with complete common mesentery malrotation are even rarer [6]; this unusual location is responsible for its delayed diagnosis and can present with serious complications. Modern imaging technics including doppler-ultrasonography and CT may reveal a transposition of the mesenteric vessels and the location of the small intestine in relation to the colon, allowing for early diagnosis [7] [8].

Laparoscopy stands out as the primary surgical management as it presents two advantages: specify the anatomical abnormality in question and then allows treatment by laparoscopic appendectomy. [9]

\section{Conclusion}

While appendicitis is by far the most frequently encountered surgical emergency in the operating room, its preoperative diagnosis, when the presentation is atypical, requires appropriate imaging notably CT and a good knowledge of the anatomical variability of the digestive tract. 


\section{References}

[1] Pinto, A., Raimondo, D.D., Tuttolomondo, A., et al. (2007) An Atypical Clinical Presentation of Acute Appendicitis in a Young Man with Midgut Malrotation. Radiography, 13, 164-168. http://dx.doi.org/10.1016/j.radi.2005.10.010

[2] Powell, D.M., Othersen, H.B. and Smit, C.D. (1989) Malrotation of the Intestines in Children: The Effect of Age on Presentation and Therapy. Journal of Pediatric Surgery, 24, 777-780. http://dx.doi.org/10.1016/S0022-3468(89)80535-4

[3] Nelson, M.J. and Pesola, G.R. (2001) Left Lower Quadrant Pain of Unusual Cause. The Journal of Emergency Medicine, 20, 241-245. http://dx.doi.org/10.1016/S0736-4679(00)00316-4

[4] Akbulut, S., Caliskan, A., Ekin, A., et al. (2010) Left-Sided Acute Appendicitis with Situs Inversus Totalis: Review of 63 Published Cases and Report of Two Cases. Journal of Gastrointestinal Surgery, 14, 1422-1428. http://dx.doi.org/10.1007/s11605-010-1210-2

[5] Huang, S.M., Yao, C.C., Tsai, T.P. and Hsu, G.W. (2008) Acute Appendicitis in Situs Inversus Totalis. Journal of the American College of Surgeons, 207, 954. http://dx.doi.org/10.1016/j.jamcollsurg.2008.03.030

[6] Welte, F.J. and Grosso, M. (2007) Left-Sided Appendicitis in a Patient with Congenital Gastrointestinal Malrotation: A Case Report. Journal of Medical Case Reports, 1, 92. http://dx.doi.org/10.1186/1752-1947-1-92

[7] Israelit, S., Brook, O.R., Nira, B.R., Guralnik, L. and Hershko, D. (2009) Left-Sided Perforated Acute Appendicitis in an Adult with Midgutmalrotation: The Role of Computed Tomography. Emergency Radiology, 16, 217-218. http://dx.doi.org/10.1007/s10140-008-0746-x

[8] Perera, W.R. and Hennessy, O.F. (2010) Clinical Images-An Unusual Case of Appendicitis. The American Journal of Surgery, 199, 79-81. http://dx.doi.org/10.1016/j.amjsurg.2009.08.047

[9] Golash, V. (2006) Laparoscopic Management of Acute Appendicitis in Situs Inversus. Journal of Minimal Access Surgery, 2, 220-221. 